\title{
Nonnegative Matrix Equations Having Positive Solutions
}

\author{
By Jerry A. Walters
}

\begin{abstract}
Suppose $\tilde{A}$ is a nonnegative invertible matrix with a positive diagonal $D=$ $\operatorname{Diag}(\tilde{A})>0$ and $\tilde{y}>0$ is a positive vector. Let $A=D^{-1} \tilde{A}$ and $y=D^{-1} \tilde{y}$. If $0<2 y-$ $A y$, then $2 y-A y \leqq x \leqq y$, where $x=A^{-1} y$.
\end{abstract}

Introduction. The inverse $A^{-1}$ of a given nonnegative invertible matrix, $A$, will usually contain negative elements; and hence for some $y>0$ the solution vector $x=A^{-1} y$ will have negative components. As suggested in the abstract there is no loss in generality in assuming Diag $(A)=I$. The condition

$$
0<2 y-A y
$$

will be shown to imply $0<x=A^{-1} y$ and to imply that $A$ is diagonally similar to the diagonally dominant matrix $Y^{-1} A Y$.

Theorem. Suppose $\tilde{A}$ is a nonnegative invertible matrix with a positive diagonal $D=\operatorname{Diag}(\tilde{A})>0$ and $\tilde{y}>0$ is a positive vector. Let $A=D^{-1} \tilde{A}$ and $y=D^{-1} \tilde{y}$. If $0<2 y-A y$, then $2 y-A y \leqq x \leqq y$, where $x=A^{-1} y$.

Proof. Let $B=A-I$ then (1) implies $0<(I-B) y$. We wish to show $2 y-$ $A y \leqq x \leqq y$, i.e. $(I-B) y \leqq(I+B)^{-1} y \leqq y$, i.e. $(I-B) y \leqq\left(I-B^{2}\right)^{-1}(I-B) y$ $\leqq y$. Let $u$ be the positive vector $u=(I-B) y$. We wish to show $u \leqq\left(I-B^{2}\right)^{-1} u$ $\leqq(I-B)^{-1} u$ which will hold provided $\left(I-B^{2}\right)^{-1}$ and $(I-B)^{-1}$ are nonnegative matrices.

These matrices will be nonnegative provided the corresponding matrix series converge, since

$$
\begin{array}{r}
I \leqq I+B^{2}+B^{4}+\cdots \leqq I+B+B^{2}+\cdots \\
\quad \text { implies } I \leqq\left(I-B^{2}\right)^{-1} \leqq(I-B)^{-1} .
\end{array}
$$

And the series will converge provided the spectral radius of $B$ satisfies $\rho(B)<1$. To see that $\rho(B)<1$, we let $y=Y e$ where $e$ is the vector having all its components equal to 1 and $Y$ is the diagonal matrix corresponding to $y$. Then, $0<(I-B) y$ implies $Y^{-1} B Y e<e$ which implies $\rho(B)=\rho\left(Y^{-1} B Y\right)<1$.

Corollary. The inequality $Y^{-1} B Y e<$ e also implies that the matrix $\left(I+Y^{-1} B Y\right)$ $=Y^{-1}(I+B) Y=Y^{-1} A Y$ is diagonally dominant.

Acknowledgment. The author is indebted to the referee for his help.

U.S.N. Radiation Laboratory

San Francisco, California 94135

Lawrence Radiation Laboratory

Berkeley, California 94709

Received September 24, 1968, revised February 3, 1969. 